\title{
Improvement to Gene Editing in Neospora Caninum. Knockout ku80 to Increase the Efficiency of Homologous Recombination
}

\section{Kaijian Wu}

China Agricultural University

\section{Xingju Song}

China Agricultural University

\section{Yayun Wu}

China Agricultural University

\section{Xu Yang}

China Agricultural University

Jing Liu

China Agricultural University

Qun Liu ( $\nabla$ qunliu@cau.edu.cn )

China Agricultural University

\section{Research Article}

Keywords: Neospora caninum, ku80, Knockout, Gene Editing $\rrbracket$

Posted Date: August 17th, 2021

DOl: https://doi.org/10.21203/rs.3.rs-800882/v1

License: (1) This work is licensed under a Creative Commons Attribution 4.0 International License. Read Full License 


\section{Abstract}

Background: The CRISPR/Cas9 technology based on homologous recombination has been widely used for gene editing in organism. Gene deletion of ku80 complex can increase the efficiency of homologous recombination(HR), or gene editing by CRISPR/Cas9 in Arabidopsis thaliana and Toxoplasma gondii, which has remained elusive in Neospora caninum.

Methods: Here, we knocked out the ku80 in Nc1 strain, named $\triangle$ ku80, using CRISPR/Cas9. In vitro, phenotype assays, including plaque formation, invasion, replication and egress were carried out to determination of the growth of $\Delta k u 80$. In vivo, we respectively injected BALB/c mice with different doses of NcKU80 and Nc1, and measured the survival curves and parasites burdens of mice infected. To test the efficiency of HR, $\Delta k u 80$ and $\mathrm{Nc1}$ strains were transfected with the same HA-tagged plasmids, and the percentage of parasites with HA-tagged was investigated.

Results: It is showed that the growth and virulence of $\Delta k u 80$ kept unaffected, while the efficiency of gene targeting via double-crossover at several genetic loci was increased 2 to 3 fold in $\Delta k u 80$.

Conclusions: It is concluded that $\Delta k u 80$ can be used as an effective strain for rapid gene editing in $N$. caninum.

\section{Background}

The global prevalence of Neosporosis poses serious harm, causing abortion in cattle, neuromuscular disorder in calves, neuromuscular injury and hind limb paralysis in puppies. Its causative pathogen is Neospora caninum, an obligate intracellular apicomplexan parasite, which is capable of parasitizing nucleated cells of legion mammalian species. $N$. caninum is highly similar to Toxoplasma gondii in life cycle, morphological and biological characteristics.

To prevent and control Neosporosis, in-deep analysis $N$. caninum gene function study closely associated with gene editing technology is required. However, most gene editing methods have common problems in practice application, such as off-target binding and low efficiency of spontaneous homologous recombination. This is also an existing issue in $N$. caninum'study, which is the reason why numerous researches focus on technological optimization. Lei et al. constructed the overexpressing strain of NcROP18, but failed to knock out it [1]. Ma et al. completely knocked out ROP5 and ROP16 genes of $N$. caninum using homologous recombination, although the screening efficiency was only $1.4 \%[2,3]$. In recent years, CRISPR/Cas9 technology has become the first choice of genome editing as a transformative DNA genome editing system. Arranz-Solís and Yang successively applied CRISPR/Cas 9 technology in $N$. caninum to improve the effectiveness of genome editing successfully, promoting the study of this parasite $[4,5]$. In present, several studies have applied CRISPR/Cas9 technology to disrupt gene in $N$. caninum. 
The CRISPR/Cas9 nuclease complex consists of guide sgRNA and Cas9 endonuclease. Cas9 can generate DNA double-strand breaks (DSB) at a specific target gene site under sgRNA guidance. Once a DSB is produced, it must be repaired because a single unrepaired DSB can result in cell death. Three pathways contribute to DSB repair in eukaryotes: homologous recombination(HDR or HR), classical nonhomologous end joining (c-NHEJ) and alternative end joining (alt-NHEJ). Among these,HR is the primary DSB repair pathway in yeasts and prokaryotes, while higher eukaryotes including $N$. caninum use c-NHEJ priorly. However, CRISPR/Cas9 technology is based on HR, which generates gene insertion and point mutations by rearranging DNA sequences in the presence of homologous DNA flank sequences. These factors lead to the low efficiency of CRISPR/Cas9 gene editing in $N$. caninum [6, 7]. To improve the HR efficiency of CRISPR/Cas9 technology in $N$. caninum, we committed to block c-NHEJ.

KU protein binds on DSB ends to initiate c-NHEJ. Studies have shown that knocking out ku80 significantly improves the efficiency of gene editing in Toxoplasma gondii without changing its biological peculiarities $[8,9]$. In this study, ku80 knockout strain of $N$. caninum was constructed to inhibit c-NHEJ using CRISPR/Cas9 technology, and the probability of HR during DNA repair was increased.

\section{Methods}

\section{Cell and Parasites Culture}

Vero cells and HFF cell lines were transduced and cultured with $6 \%$ and $10 \%$ fetal bovine serum (FBS, Gibco, USA) DMEM (Dulbecco's Modified Eagle's Medium). N.caninum was cultured in the above two cells with $1 \%$ fetal bovine serum DMEM. The culture conditions are $37^{\circ} \mathrm{C}$ and $5 \% \mathrm{CO}_{2}$.

\section{Plasmid and Transgenic Parasite Construction}

Primers for PCR amplification are shown in Table 1. The gRNA targeting site for NcKU80 (5'GTTCAGCCTTCAGCTCTCCG-3') was designed in the EuPaGDT Library of ToxoDB, and pCRISPR-Cas9NcKU80 was constructed based on PSAG1-Cas9-NLS-GFP-SAG1 plasmid preserved in the Key Laboratory of Animal Parasitology (Beijing, China). The method is as described by Yang [5]. The gRNA was intergrated into the plasmid downstream of the 3 'Flank sequence of the U6 promoter by ligation transformation (Vazyme, Nanjing, China).

The knockout plasmid was constructed on the basis of plasmid pTCR-CD retained in the laboratory. $5^{\prime}$ and 3 ' homologous flanks of NcKU80 were amplified using Nc1 genome as a template. To facilitate screening, the chloramphenicol resistance gene and red fluorescent protein gene (RFP) were inserted between $5^{\prime}$ and $3^{\prime}$ homologous flanks, and ligated into plasmid backbone PTCR-CD with flanks. The plasmid CRISPR and the plasmid pTCR-NcKU80 were co-transfected into Nc1. And used the limited dilution method to cloned Nc $\Delta$ ku 80 after chloramphenicol treatment.

The lab-built plasmid CRISPR and pTCR of NcGrxC5-oe, NcGrxC5-HA, NcGrxS14-oe, NcGrxS14-HA was constructed by the method as above. The difference was that these PTCR plasmids were integrated the 
DHFR gene inserted and the strains were treated with pyrimethamine after co-transfect.

\section{Plaque Assay}

200 tachyzoites were added into 12-well plate HFF cells and fixed with 4\% paraformaldehyde after 8 days, and then stained with $0.2 \%$ crystal violet dye. The plaque area was calculated using Photoshop CC 2018 software (Adobe, United States). The calculation was repeated 3 times to take the average. Three independent trials were carried out.

\section{Immunofluorescence Assay}

The 12-well plates were fixed with $4 \%$ paraformaldehyde, permeated with $0.25 \%$ Triton X-100 for 20 minutes and were blocked with $3 \% \mathrm{BSA}$ for 30 minutes. sealed with $3 \% \mathrm{BSA}$. The primary antibodies were rabbit anti-SRS2 (1:200) and mouse anti-HA (Sigma-Aldrich, Saint Louis, MO, USA; 1:100). While the secondary antibodies were the goat anti-mouse IgG1 (FITC-labeled) and goat anti-rabbit IgG1 (CY3labeled). And nucleus was labelled by DAPI. All antibodies were incubated for 1 hour.

\section{Invasion assay}

A total of $1 \times 10^{5}$ tachyzoites were added to the HFF cell that cultured on 12-well plates. After 1 hour, the parasites that didn't attach or invade to cells were washed with PBS. Immunofluorescence assay was performed after cultured for 24 hours. The 12-well plates were observed using a fluorescence microscope, and the number of HFF cells and PV were calculated by randomly selecting 5 visual fields from the top, bottom, left, right and middle positions respectively. The invasion capacity of the parasite is the number of PV in the field divided by the number of HFF cells. The calculation was repeated 3 times to take the average. Three independent trials were carried out.

\section{Replication assay}

Cultivate parasites and IFA in the same way as invasion assay. 100 PVs were randomly calculated using a fluorescence microscope, and then the number of PVs containing different amounts of tachyzoites was calculated. The calculation was repeated 3 times to take the average. Three independent trials were carried out.

\section{Egress assay}

Cultivate parasites in the same way as invasion assay and treated with calcium ionophore A23187 (Sigma-Aldrich, USA) for 3 minutes after 24 hours. IFA was then utilized. The escaping ability of tachyzoites is expressed as the proportion of escaping PVs to the total PVs. Calculate 100 PVs and the calculation was repeated 3 times to take the average. Three independent trials were carried out.

\section{Virulence tests in mice}

The 6-week-old female BALB/c mice were purchased from Jinmuyang Laboratory Animal Breeding Co., Ltd. (Beijing, China) and were kept in a sterile cage for a week, ad libitum access to clean food and water. 
Five per group mice were intraperitoneally injected with different doses of tachyzoites and were observed for 30 days.

\section{Quantitative Real-Time PCR}

Parasite quantitative detection in mice by qPCR was carried out following the method CollantesFernández reported [10]. The mice were humanely sacrificed at 30 days, then collected brains and extracted DNA using the DNA Extraction Kit (Beijing Aide Lai Biotechnology Co. Ltd., Beijing, China). Primers were designed to quantitatively detect 28S RNA of mice and Nc5 gene of N.caninum by qPCR. Established the standard curve of 28S RNA and Nc5. The parasite load was expressed as the content of N.caninum Nc5 per nanogram of mouse $28 \mathrm{~S}$ rRNA.

\section{Data Analysis}

All data were analyzed using GraphPad Prism 8.0.2 (San Diego, CA, USA), and the results were displayed as mean \pm standard deviation and tested by two-way ANOVA and the Student's $t$ test. $P$-values are indicated as asterisks: $* p<0.05, * * p<0.01, * * * p<0.001$ and $* * * * p<0.0001$. We consider all $p<0.05$ to be significant.

\section{Results}

Deletion of ku80 in N. caninum

The KU domain-containing protein of the $N$. caninum was searched in the ToxoDB genomic resource database, and two genes were found (NCLIV_056045 and NCLIV_064150). BLASTp analysis of these two genes showed that NCLIV_056045 had a higher percent identity with KU80 protein of Arabidopsis and Toxoplasma gondii, respectively $24.06 \%$ and $64.81 \%$ (E-value of 3e-10 and 0.0), named Ncku80.

The CRISPR/Cas9 technology platform constructed by Yang et al. [5] was used to delete ku80 in the $N$. caninum wild-type (WT) strain (Nc1) parasites (Fig. 1A). Identification of ku80 genewas carried out using PCR. Two primer pairs on the upstream and downstream of ku80 gene were designed to identify the absence of the target gene. Further, two primer pairs were designed to verify the integration in 5 'flank and 3'flank (Fig. 1B). Two gene-deletion strains, named $\Delta k u 80$, were obtained by screening. A red fluorescent protein (RFP) coding sequence was added to the $5^{\prime}$ end of the chloramphenicol acetyltransferase (CAT) gene, fluorescent knockout strains were captured with an inverted fluorescence microscope (Fig. 1C).

\section{Knocking out ku80 did not influence the phenotype of $\mathrm{N}$. caninum in vitro}

The ideal result of this study is to construct a parent strain that can be used in practical study. Therefore, in addition to increase the efficiency of HR, the morphological, growth rate and virulence of the constructed strain cannot be changed. In terms of morphological characteristics, $\Delta k u 80$ and Nc1 were observed under optical microscope and fluorescence microscope after IFA. Both $\Delta k u 80$ and Nc1 strains can form elliptic parasitophorous vacuole (PV), and the tachyzoites have a classical crescent-shaped the same as Nc1(Fig. 2A). 
In terms of growth, $N$. caninum has a complete lytic cycle in which the tachyzoite first invades the host cell, and the tachyzoites maintained proliferating in the host cell until sufficiently large PV is produced[11]. Subsequently, the PV bursts, and the released tachyzoites invaded other cells. In order to analyze whether knocking out KU80 affects $N$. caninum' lytic cycles and virulence, phenotype assays, including plaque formation, invasion, replication and egress should be carried out. Plaque formation assays can comprehensively reflect the parasites develop ability in vitro. Both $\mathrm{Nc} 1$ and $\Delta k u 80$ led to uniform, clear morphological rules and plaques (Fig. 2B). We randomly selected 50 plaques and calculated their area. T-test analysis showed that $\Delta k u 80$ kept the parallel capability of plaque formation from its parental strain. ( $P=0.4029$, Fig. 2 C).

For invasion assay, IFA showed $\Delta k u 80$ invade to HFF cells normally, which suggests there is no barrier or delay to invasion in the knockout strain $(P=0.3478$, Fig. 2D). For replication assay, 100 vacuoles were randomly selected to calculate the number of tachyzoites inside each vacuole. There were no difference between Nc1 and $\Delta k u 80$ on the numbers of vacuoles carrying 2, 4, 8, 16 or more tachyzoites $(P=0.9814$, Fig. 2D). For the egress assay, Nc1 and $\Delta k u 80$ were stimulated with the calcium ionophore A23187 at the same time. It was observed that $\Delta k u 80$ had similar speed of escaping from HFF cells as Nc1. $(P=0.2748$, Fig. 2D). Overall, the above experiments proved that compared with $\mathrm{Nc1}$, knocking out NcKU80 had no effect on the $N$. caninum phenotype in vitro.

\section{Knocking out ku80 did not influence the virulence in mice of $\mathrm{N}$. caninum}

To estimate the effect of knocking out ku80 on the virulence of $N$. caninum in mice, we respectively injected BALB/c mice with different doses of $\Delta k u 80$ and Nc1. With the injection of $1.5 \times 10^{7}$ tachyzoites, it was found that two of the five mice died on 7dpi and one died on 10dpi in Nc1 group. Similarly, five mice have two deaths on $7 \mathrm{dpi}$ and one death on $18 \mathrm{dpi}$ in $\triangle k u 80$ group. Statistical analysis using Graph Pad 8.0 found no significant difference between the two groups $(P=0.9119$, Fig. $3 \mathrm{~A})$. With the injection of $2 \mathrm{x}$ $10^{7}$ tachyzoites, two of five mice respectively died on $7 \mathrm{dpi}$ and $13 \mathrm{dpi}$ in $\Delta k u 80$ group. While three of the five mice respectively died on days 7, 9, and 18 in Nc1 group. Statistical analysis demonstrated that the two groups also share the same survival status after injection with same doses $(P=0.6576$, Fig. 3B). The mice injected with $1 \times 10^{7}$ tachyzoites in the two groups survived 30 days of continuous observation. On the 31 st day, brain tissues of the surviving mice were collected for qPCR to detect the parasite burden in the brain, and the result showed there was no difference in the parasite burden between the two groups $(P=0.1363$, Fig. 3C).

Results 2 and 3 illustrated that the growth and the virulence of $\Delta k u 80$ kept unaffected.

\section{Comparison of homologous recombination efficiency between $\Delta \mathrm{ku} 80$ and Nc1}

Since KU80 plays an important role in NHEJ, it is hypothesized that knocking out $\Delta k u 80$ can block NHEJ, thus increasing the probability of HR. In the studies of $N$. caninum, overexpression and mutation are the main editing technology. The efficiency of the gene replacement events in the ku80 knockout background can be evaluated by introducing HA-tag into plasmids permits. The HA-tagged mutation (HA) and 
overexpression (oe) plasmids of glutaredoxins C5(GrxC5) and S14(GrxS14) were constructed previously. Previous experiments had shown apicoplast localization of GrxC5 and GrxS14, and neither of their overexpression nor gene deletion affected the growth of the $N$. caninum (unpublished data). Therefore, to determine if the gene replacement efficiency is increased in the $k u 80$ knockout background, we chose the lab-built HA-tagged overexpression to targeted into UPRT locus, and HA-tagged mutation plasmids to GrxC5 and GrxS14 locus. $1 \times 10^{7} \Delta k u 80$ and Nc1 tachyzoites were transfected with an equal volume of HA-tagged plasmid and CRISPR/Cas9 plasmid at the same time. All strains (NcGrxC5-oe, NcGrxC5-HA, NcGrXS14-oe, NcGrXS14-HA) were verified by PCR for the insertion of endogenous HA-tags correctly. IFA was used to measure the percentage of HA-tagged in 300-tachyzoites after 10 days of cultivation (Fig. 4A). Each plasmid was transfected 5 times.

The statistical results showed that the gene replacement events of $\Delta k u 80$ was remarkably increased more than twice or even three times that of Nc1, showing a significant difference (Fig. 4B-4E). It is worth noting that using $\Delta k u 80$ as parent strains success $90.75 \%$ gene replace, while that is $63.25 \%$ in Nc1, although no significant difference was found in the two groups (Fig. $4 \mathrm{~F}, P=0.0678$ ). Above all, our results proved that the knockout of ku80 could significantly increase the efficiency of HR in N.caninum.

\section{Discussion}

In this study, Ncku80 knockout strain was developed using CRISPR/Cas9 technology, and the phenotype and virulence experiment indicated that the deletion of $N c k u 80$ had no significant impact on the growth or the virulence of N.caninum. Since KU80 is an important factor of c-NHEJ DNA repair pathway, the frequency of the gene replacement events of $\Delta k u 80$ was explored by transfecting HA-tagged plasmids. It is proved that the efficiency of HR in $\Delta k u 80$ has raise considerably. Therefore, this strain can be applied to the study of N.caninum to advance the efficiency of gene edition.

CRISPR/Cas9 technology has been introduced into the apicomplexan parasite, such as Toxoplasma, Plasmodium, Cryptosporidium[12] as well as N. caninum, which has considerably promoted the study on these parasites. However, due to the priority of c-NHEJ, the efficiency of HR is relatively low when CRISPR/Cas 9 is used for gene insertion or deletion in many organisms. That is the reason why researchers attempted to construct $k u$ gene deletion strains, and some results have been achieved. $k u$ gene homologous sequence deletion strains have been constructed in various fungi [13] by blocking cNHEJ, the efficiency of HR was increased to nearly $100 \%$. In Arabidopsis, mutations in ku70 can result high-frequency gene targeting [14]. In Toxoplasma, ku80 gene deletion strains have also been constructed, markedly increased gene replacement efficiency to 300 to 400 fold compared to wild-type strains[9]. While little efforts were made on ku80 knockout strain in $N$. caninum. We successfully filled in this gap, and proved the efficiency of HR was significantly increased in $\Delta k u 80$.

The efficiency of CRISPR/Cas9 technology has always been an obstacle[15]. Several parameters affect the efficiency of gene targeting in CRISPR/Cas9 technology, including the fitness score of the target gene, drug selectable markers, plasmid, the targeting DNA flank lengths, the life force of parasite and so on. 
Some methods have been reported to recover the defect, including substituting the $N$. caninum specific promoter and terminator $[5,15]$. In this study, deletion of ku80 was proved to work well.

Although KU70/ KU80-mediated c-NHEJ plays a role in DSB repair in many protozoa, the c-NHEJ factor is often lost in parasites[16]. For example, in Plasmodium, Trypanosoma brucei, and African trypanosomes, the repair of DSB is mediated by a Ku-independent microhomology-mediated end Joining (MMEJ) [1719]. While in $N$. caninum, three conserved factors of c-HNFJ were retained: KU70, KU80 and Lig4[16]. Our study confirmed the existence of KU80 protein in N. caninum, and it was revealed that the deletion of $\Delta k u 80$ decreases the rate of c-NHEJ, thus increasing HR's. An important issue is the virulence of $\Delta k u 80$, we determined that $\triangle k u 80$ can invade into the host cell, multiply in and release from it, pass across the blood-brain barrier and cause a same injury in the mice as Nc1. It means ku80 knockout background did not lead to develop defect in N. caninum, which are similar to fungi and Toxoplasma[9, 13].

Constructing $\Delta k u 80$ without any drug tagging has been tried originally but ended in failure. In order to broaden the application range of this strain, the knockout of the chloramphenicol acetyltransferase gene is underway. It may be easier to successfully generate in the $k u 80$ knockout background. When using this strain for gene editing, selectable markers other than chloramphenicol could be used for positive or negative selection. Positive selection including DHFR (resistance to pyrimethamine), HXGPRT (resistance to Mycophenoloic acid/xanthine), while negative selection including UPRT (resistance to 5fluorodeoxyuracil), SNFR1 (resistance to Sinefungin) and HXGPRT (resistance to 6-thioxanthine) [20].

When four plasmids were used for transfecting in the ku80 knockout background, the success rate of gene replacement was unchanged, additionally the efficiency was more than doubled. It suggested that HR might play a less important role in the success rate of transfection than other factors, such as the target gene locus, the mass of the plasmid and drug screening marker. The dramatic improvement of HR efficiency in $\Delta k u 80$ makes it easier to screen the monoclonal strains. Moreover, the success rate of overexpression was higher than that of mutation, which may be due to different drug markers used (floxuridine, FUDR in oe and DHFR in HA). It demonstrated the value of pharmacological markers in gene editing.

\section{Conclusions}

A strain was constructed with ku80 deletion, which improved the efficiency of homologous recombination and could improve the efficiency of gene editing in the study of N.caninum.

\section{Abbreviations}

HR

homologous recombination, c-NHEJ:classical nonhomologous end joining (c-NHEJ), DSB:DNA doublestrand breaks, PCR:polymerase chain reaction, IFA:immunofluorescence Assay, qPCR:Quantitative RealTime PCR, Vero cells:African green monkey kidney cell, HFF:human foreskin fibroblast, 
PV:parasitophorous vacuole, HA:hemagglutinin, oe:overexpression, FUDR:floxuridine, CAT:chloramphenicol acetyltransferase, DHFR:dihydrofolate reductase, BSA:bovine albumin, PBS:phosphate buffered saline, FITC:fluorescein isothiocyanate isomer, DAPI:2-(4-Amidinophenyl)-6indolecarbamidine dihydrochloride

\section{Declarations}

\section{Ethics approval and consent to participate}

The raising and handling of animals are in compliance with the recommendations in the Guide for the Care and Use of Laboratory Animals in China. All experiments were approved by the Institutional Animal Care and Use Committee of China Agricultural University (under the certificate of Beijing Laboratory Animal employee ID: 18049). The surviving mice infected with $1 \times 10^{7}$ doses were injected with atropine $(0.02 \mathrm{mg} / \mathrm{kg})$ at $30 \mathrm{dpi}$, and were humanely sacrificed by cervical dislocation.

\section{Consent for publication}

Not applicable

\section{Availability of data and materials}

Data supporting the conclusions of this article are included within the article.

\section{Competing interests}

The authors declare that the research was conducted without conflict of interest.

\section{Author' contributions}

QL and $\mathrm{JL}$ conceived the study. KW performed the experiments. QL and $\mathrm{KW}$ analyzed the data and drafted the manuscript. XS helped in animal experiments. YW and $X Y$ helped in manuscript writing. All authors read and approved the final manuscript.

\section{Acknowledgements}

We are appreciation to Prof. Shaojun Long (China Agricultural University, China) for his confocal microscopy. We are also grateful to Prof. Bang Shen (Huazhong Agricultural University, China) for providing the CRISPR/CAS9 vector.

\section{References}

1. Lei T, Wang H, Liu J, Nan H, Liu Q. ROP18 is a key factor responsible for virulence difference between Toxoplasma gondii and Neospora caninum. PLoS One. 2014;9:e99744. 
2. Ma L, Liu J, Li M, Fu Y, Zhang X, Liu Q. Rhoptry protein 5 (ROP5) Is a key virulence factor in Neospora caninum. Front Microbiol. 2017;8:370.

3. Ma L, Liu G, Liu J, Li M, Zhang H, Tang D, et al. Neospora caninum ROP16 play an important role in the pathogenicity by phosphorylating host cell STAT3. Vet Parasitol. 2017;243:135-47.

4. Arranz-Solís D, Regidor-Cerrillo J, Lourido S, Ortega-Mora LM, Saeij JPJ. Toxoplasma. CRISPR/Cas9 constructs are functional for gene disruption in Neospora caninum. Int J Parasitol. 2018;48:597600.

5. Yang C, Liu J, Ma L, Zhang X, Zhang X, Zhou B, et al. NcGRA17 is an important regulator of parasitophorous vacuole morphology and pathogenicity of Neospora caninum. Vet Parasitol. 2018;264:26-34.

6. Hsu PD, Lander ES, Zhang F. Development and applications of CRISPR-Cas9 for genome engineering. Cell. 2014;157:1262-78.

7. Canver MC, Bauer DE, Dass A, Yien YY, Chung J, Masuda T, et al. Characterization of genomic deletion efficiency mediated by clustered regularly interspaced short palindromic repeats (CRISPR)/Cas9 nuclease system in mammalian cells. J Biol Chem. 2017;292:2556.

8. Fox BA, Falla A, Rommereim LM, Tomita T, Gigley JP, Mercier C, et al. Type II Toxoplasma gondii KU80 knockout strains enable functional analysis of genes required for cyst development and latent infection. Eukaryot Cell. 2011;10:1193-206.

9. Huynh M, Carruthers VB. Tagging of endogenous genes in a Toxoplasma gondii strain lacking Ku80. Eukaryotic cell.2009; 8: 530-539.

10. Collantes-Fernández E, Zaballos A, Alvarez-García G, Ortega-Mora LM. Quantitative detection of Neospora caninum in bovine aborted fetuses and experimentally infected mice by real-time PCR. J Clin Microbiol. 2002;40:1194-8.

11. Blader IJ, Coleman BI, Chen C, Gubbels M. Lytic Cycle of Toxoplasma gondii: 15 Years Later. Annu Rev Microbiol. 2015;69:463-85.

12. Suarez CE, Bishop RP, Alzan HF, Poole WA, Cooke BM. Advances in the application of genetic manipulation methods to apicomplexan parasites. Int J Parasitol. 2017;47:701-10.

13. Goins CL, Gerik KJ, Lodge JK. Improvements to gene deletion in the fungal pathogen Cryptococcus neoformans: absence of Ku proteins increases homologous recombination, and co-transformation of independent DNA molecules allows rapid complementation of deletion phenotypes. FG B. 2006;43:531-44.

14. Naguleswaran A, Elias EV, McClintick J, Edenberg HJ, Sullivan WJ. Toxoplasma gondii lysine acetyltransferase GCN5-A functions in the cellular response to alkaline stress and expression of cyst genes. PLoS Pathog. 2010;6:e1001232.

15. Zhao P, Zhang N, Dong J, Li J, Wang X, Li X, et al. Effects of Dense Granular Protein 6 (GRA6) Disruption on Virulence. Front Vet Sci. 2020;7:562730.

16. Nenarokova A, Záhonová K, Krasilnikova M, Gahura O, McCulloch R, Zíková A, et al. Causes and effects of loss of classical nonhomologous end joining pathway in parasitic eukaryotes.MBio.2019; 
10.

17. Xu R, Liu Y, Fan R, Liang R, Yue L, Liu S, et al. Generation and functional characterisation of Plasmodium yoelii csp deletion mutants using a microhomology-based CRISPR/Cas9 method. Int $\mathrm{J}$ Parasitol. 2019;49:705-14.

18. Burton P, McBride DJ, Wilkes JM, Barry JD, McCulloch R. Ku heterodimer-independent end joining in Trypanosoma brucei cell extracts relies upon sequence microhomology. Eukaryot Cell. 2007;6:177381.

19. Glover L, McCulloch R, Horn D. Sequence homology and microhomology dominate chromosomal double-strand break repair in African trypanosomes. Nucleic Acids Res. 2008;36:2608-18.

20. Shen B, Brown K, Long S, Sibley LD. Development of CRISPR/Cas9 for efficient genome editing in Toxoplasma gondii. Methods Mol Biol.2017; 1498.

\section{Figures}


a

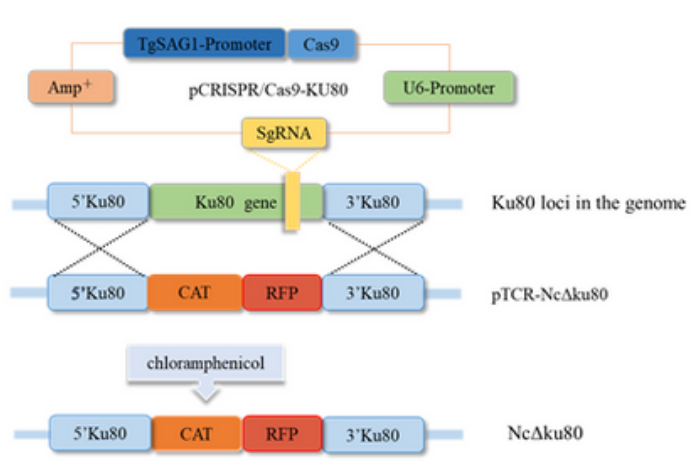

c

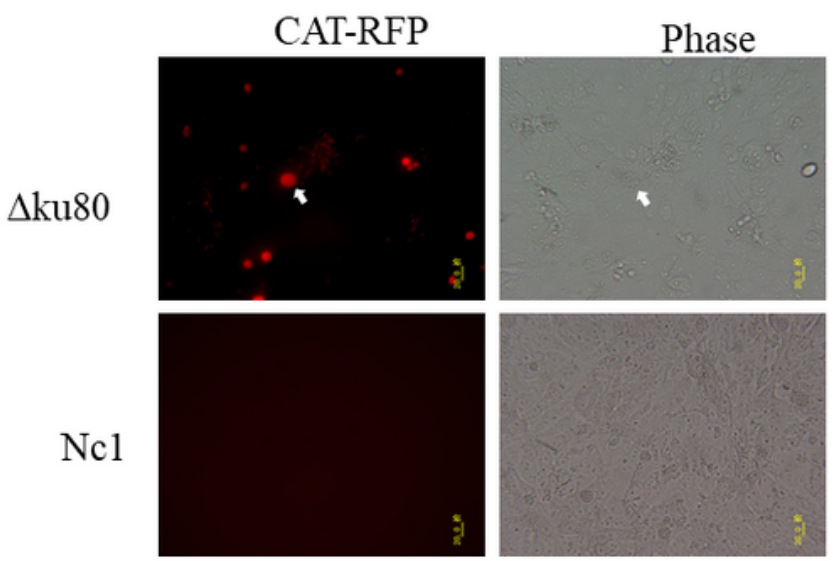

b



F3/R3

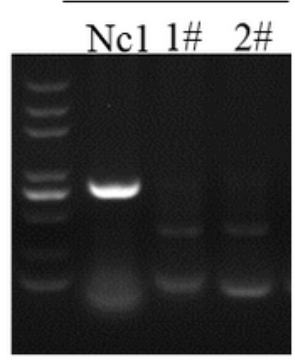

$\mathrm{F} 2 / \mathrm{R} 2$



F4/R4

Nc1 1\# 2\#



Figl:Deletion of ku80 gene in $N$. caninum

A graphical representation of substitute $k u 80$ gene with a chloramphenicol resistance gene(a). The gRNA targeting site for Ncku80 (GTTCAGCCTTCAGCTCTCCG) was designed in the EuPaGDT Library of ToxoDB. The recombinant plasmid includes a chloramphenicol resistance gene that was inserted between 5' and 3' homologous flanks of Ncku80.The deletion of $k u 80$ gene and the chimerism of CAT were identified(b). Primers F1/R1 and F2/R2 were designed to verify the integration in 5'flank and 3'flank.Primers F3/R3 and F4/R4 were designed to identify the deletion of the $k u 80$ gene.The recombinant plasmid contained a weak red fluorescence, which is trailed off after subculture(c).

\section{Figure 1}

See image above for figure legend 
a

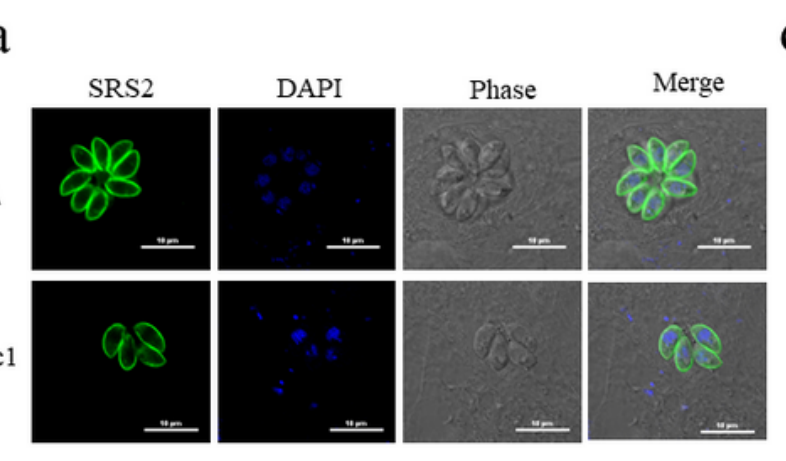

b

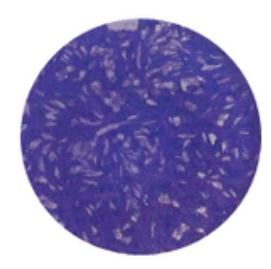

$\mathrm{Ncl}$

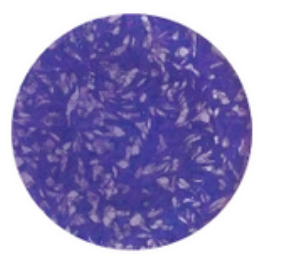

$\mathrm{Nc} \Delta \mathrm{KU} 80$

d



e

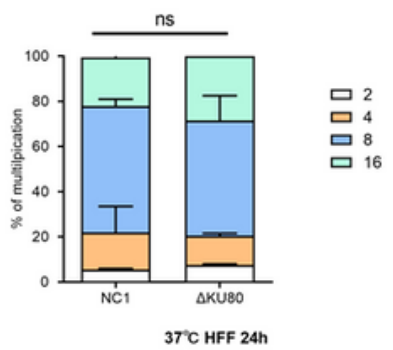

$\mathrm{c}$


Fig2: Determination of the phenotype of $\triangle k u 80$ in vitro.

IFA using anti-NcSRS2 antibodies was performed to investigate the morphological characteristics of $\Delta k u 80$ (a). Plaque formation assays(b).50 plaques were randomly selected and their area were calculated by Photoshop CC software (Adobe, United States) using Pixel point. The statistical test used was $t$-test(c). Invasion assay (d), Replication assay (e) and Egress assay (f) showed no significant differences between $\Delta k u 80$ and $\mathrm{Ncl}$ in the ability of growth. The statistical test used in three assay was $t$-test, two-way anova and $t$-test separately (d). Three independent trials were carried out in each assay. ns $=$ no significant difference.

\section{Figure 2}

See image above for figure legend 
a

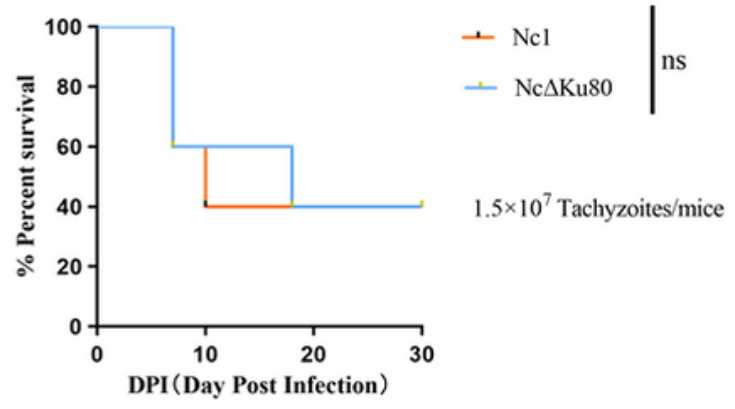

$\mathrm{C}$

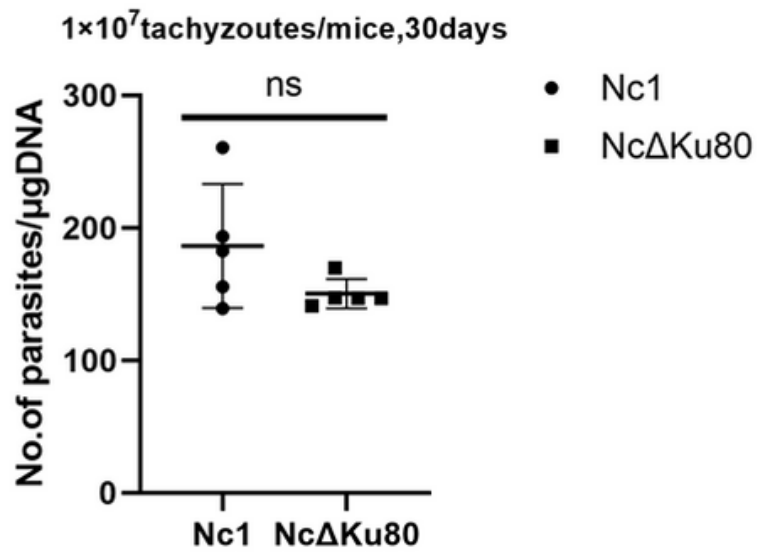

$\mathrm{b}$

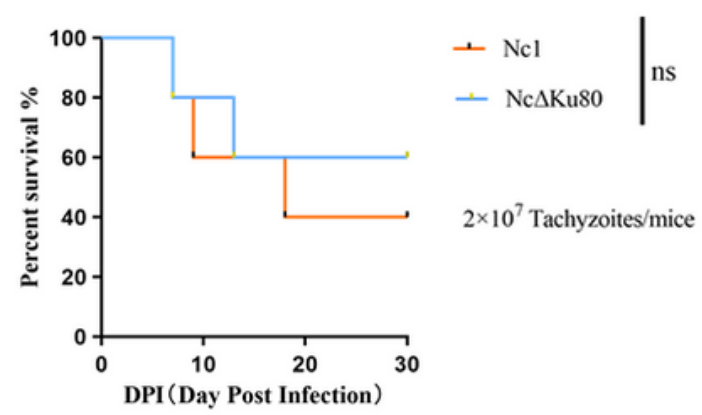

Fig3: Determination of the virulence of $4 k u 80$ in mice.

Growth curve of BALB/c mice injected with $1.5 \times 10^{7}$ (a) and $2 \times 10^{7}(\mathrm{~b})$ doses of tachyzoites. qPCR was used to detect the parasite burden in the brain of mice injected with $1.5 \times 10^{7}$ doses of tachyzoites(c). The survival curve and the Statistical analysis were performed with Graph Pad Prism (SAS Institute Inc., United States), showing no significant differences virulence between $\Delta k u 80$ and $\mathrm{Ncl}$ in mice. Data were identified by $t$-test. ns $=$ no significant difference.

\section{Figure 3}

See image above for figure legend 


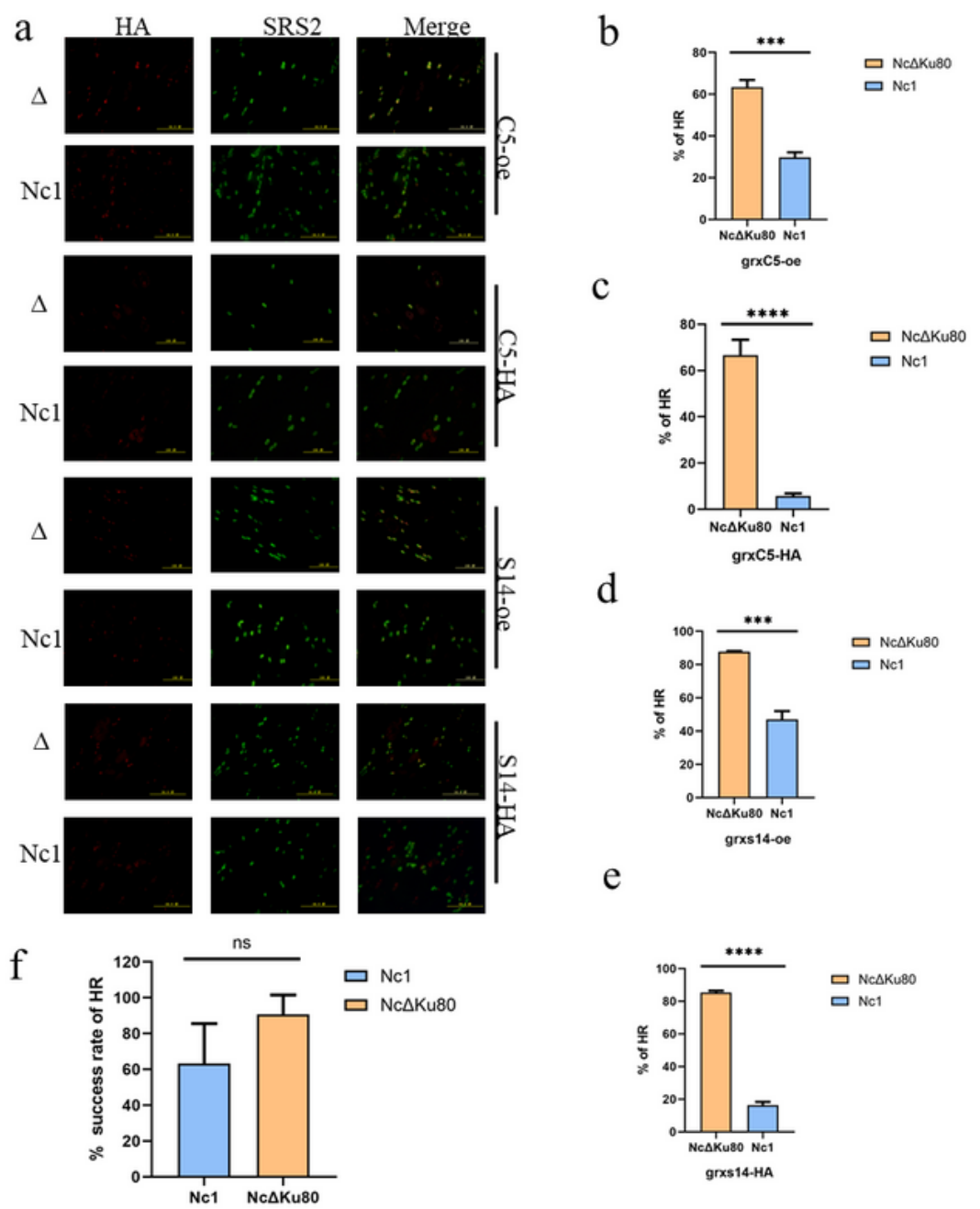

Fig4: Comparison of homologous recombination efficiency between $\triangle k u 80$ and $\mathrm{Ncl} \mathrm{s}$ $\triangle k u 80$ and $\mathrm{Ncl}$ were transfected with an equal volume of HA-tagged plasmid(a)(b: NcGrxC5-oe; c:NcGrxC5-HA;d:NcGrxS14-oe; e:NcGrxS14-HA) and CRISPR/Cas9 plasmid at the same time. The statistical results showed that the HR of $\Delta k u 80$ was remarkably increased. Each plasmid was transfected 5 times. f: The homologous recombination ratio in 5 transfections of 4 type of plasmids, showing no significant differences between $\Delta k u 80$ and $\mathrm{Ncl}$ background. Data were identified by $t$-test. $* * * \mathrm{p}<$ $0.001, * * * * \mathrm{p}<0.0001, \mathrm{~ns}=$ no significant difference.

\section{Figure 4}

See image above for figure legend

\section{Supplementary Files}

This is a list of supplementary files associated with this preprint. Click to download. 
- graphicalabstract.pdf

Page 16/16 